\title{
Cangxitongbi capsule protects articular cartilage of the knee in rats by regulating ADAMTS-5
}

\author{
Xu-Yu Song ${ }^{1 \#}$, Wen-Peng Xie ${ }^{2 \#}$, Peng Zhang ${ }^{1}$, Min Zhao $^{3}$, Rong-Xiu Bi ${ }^{2}$ \\ ${ }^{1}$ First Clinical College, Shandong University of Traditional Chinese Medicine, Jinan, China; ${ }^{2}$ Department of Orthopedics, Affiliated Hospital of \\ Shandong University of Traditional Chinese Medicine, Jinan, China; ${ }^{3}$ Department of Paediatrics, Shandong Maternal and Child Health Hospital, \\ Jinan, China \\ Contributions: (I) Conception and design: XY Song, WP Xie, RX Bi; (II) Administrative support: RX Bi; (III) Provision of study materials or patients: \\ XY Song, WP Xie; (IV) Collection and assembly of data: XY Song, P Zhang, M Zhao; (V) Data analysis and interpretation: P Zhang, M Zhao; (VI) \\ Manuscript writing: All authors; (VII) Final approval of manuscript: All authors. \\ ${ }^{\#}$ These authors contributed equally. \\ Correspondence to: Prof. Rong-Xiu Bi. Department of Orthopedics, Affiliated Hospital of Shandong University of Traditional Chinese Medicine, \\ Jinan, China. Email: birongxiu@hotmail.com.
}

Background: Knee osteoarthritis (KOA) is a disease with a high incidence in elderly patients and traditional Chinese medicine has a significant effect on the treatment of KOA. Cangxitongbi capsule (CXTB) is a traditional Chinese medicine for KOA treatment and has a remarkable curative effect. The purpose of this article is to investigate the mechanism of CXTB in protecting joint cartilage on KOA rats.

Methods: A total of 30 male Sprague-Dawley rats were randomly assigned into five groups: control group; model group; low-, mid-, and high-dose CXTB groups $(17.5,35$, and $70 \mathrm{mg} / \mathrm{mL})$. KOA models were made by modified Hulth method except the control group. After pharmacological administration for 4 weeks, knee articular cartilages were observed by hematoxylin and eosin (HE) staining and evaluated by Mankin score. Western blot and reverse transcription-polymerase chain reaction (RT-PCR) were used to detect the concentration of ADAMTS-5. The peripheral blood of the rats was collected to detect content of interleukin-1 beta (IL-1 $\beta$ ) and tumor necrosis factor-alpha (TNF- $\alpha$ ) by enzyme-linked immunosorbent assay (ELISA).

Results: The morphological structure of cartilage in the 3 CXTB groups was significantly improved compared with the model group, and the improvement positively correlated with the drug dosage $(\mathrm{P}<0.05)$. Compared with the model group, the expression levels of ADAMTS -5 of the 3 CXTB groups was obviously decreased $(\mathrm{P}<0.05)$. Furthermore, the upstream targets of ADAMTS-5, including IL-1 $\beta$ and TNF- $\alpha$ were down-regulated in the 3 CXTB groups $(\mathrm{P}<0.05)$.

Conclusions: Knee joint cartilage on KOA model rats is protected by CXTB via down-regulation of ADAMTS-5. The upstream targets of ADAMTS-5, IL-1 $\beta$ and TNF- $\alpha$, were also down-regulated by CXTB.

Keywords: Chinese medicine; Cangxitongbi capsule (CXTB); ADAMTS-5; articular cartilage; knee osteoarthritis (KOA)

Submitted Sep 18, 2020. Accepted for publication Oct 28, 2020.

doi: 10.21037/atm-20-7011

View this article at: http://dx.doi.org/10.21037/atm-20-7011 


\section{Introduction}

Knee osteoarthritis (KOA) is a chronic joint disease characterized by cartilage degeneration, loss, subchondral and new bone formation at the joint edge. The disease is also an important cause of joint pain and even disability in the elderly $(1,2)$. The most common symptoms of KOA include joint pain, stiffness, and swelling, which progresses to severe joint dysfunction and irreversible loss of motor function. The pathogenesis of $\mathrm{KOA}$ is multifactorial and includes joint injury, aging, and trauma $(3,4)$. The treatment of KOA includes surgery and conservative treatment. In the early stage of the disease, the main point of conservative treatment is to protect articular cartilage.

Proteoglycan is an important component of the articular cartilage matrix, of which articular cartilage is composed. The loss of proteoglycan is considered to be an important mechanism in the pathogenesis of KOA. Previous studies have discovered that a disintegrin and metalloproteinase with thrombospondin motifs-5 (ADAMTS-5) can promote the hydrolysis of polysaccharides. Conversely, a recent study showed that interleukin-1 beta (IL-1 $\beta$ ) and tumor necrosis factor-alpha (TNF- $\alpha)$ are closely related with the destruction of cartilage, and they are involved in the upregulation of ADAMTS-5 (5-7). Therefore, inhibitors of IL- $1 \beta$ and TNF- $\alpha$ could be promising targets for treating KOA.

Cangxitongbi capsule (CXTB), a Chinese herbal compound, is reported to have benefits in alleviating pain, protecting cartilage, and maintaining knee joint function. Our previous studies showed that CXTB could delay cartilage degeneration and promote cartilage repair. However, the effects of CXTB on ADAMTS-5 still remain unknown. So, in this study we established KOA rat models and explored the mechanism of CXTB action on ADAMTS-5. We also investigated the effects of CXTB on IL- $1 \beta$, TNF- $\alpha$, and the upstream targets of ADAMTS-5. This will be helpful in unveiling the potential mechanisms of Chinese medicine in treating KOA.

We present the following article in accordance with the ARRIVE reporting checklist (available at http://dx.doi. org/10.21037/atm-20-7011).

\section{Methods}

\section{Drug preparation}

A superfine powder of CXTB (Affiliated Hospital of Shandong University of Traditional Chinese Medicine
Pharmaceutical Co., Ltd., \# Z20130043, China) was dissolved in saline solution and administered at a low-dose of $17.5 \mathrm{mg} / \mathrm{mL}$, a mid-dose of $35 \mathrm{mg} / \mathrm{mL}$, and a high-dose of $70 \mathrm{mg} / \mathrm{mL}$. The solutions were stored in a refrigerator at $4{ }^{\circ} \mathrm{C}$ before intragastric administration.

\section{Animals}

A total of 30 adult male Sprague-Dawley (SD) rats [SPF grade, 4 weeks (wk) old, weighing $223 \pm 13 \mathrm{~g}$ ] were purchased from Jinan Pengyue Experimental Animal Breeding Co., Ltd [animal cert. \# SCXK(LU)20140007]. They were reared on a $12-\mathrm{h}$ light/dark schedule at $25 \pm 2{ }^{\circ} \mathrm{C}, 55 \% \pm 5 \%$ humidity, and were allowed free access to food and water. The experiments conformed to the guidelines for the care and use of laboratory animals published by the United States National Institutes of Health. All animal experiments were approved by the Animal Ethics Committee of Affiliated Hospital of Shandong University of Traditional Chinese Medicine (AWE-2019-044).

\section{Modeling and administration}

The rats were randomly assigned into five groups: control group; model group; low-, mid-, and high-dose CXTB groups. With the exception of the control group, rats were injected with $1 \%$ pentobarbital $(50 \mathrm{mg} / \mathrm{kg}$ ) for anesthesia and the left knee joint was exposed through a medial patellar approach after disinfection.

The anterior cruciate and medial collateral ligaments were transected and the medial meniscus was resected (modified Hulth method) to establish the KOA models $(8,9)$. After $4 \mathrm{wk}, \mathrm{X}$-ray film of the knee joint was taken. There were multiple hyperosteogenic, sclerotic, and cystic changes. The surface of the knee joint was rough and unsmooth, which was taken as confirmation that the model was successful (Figure 1).

The low-, mid-, and high-dose CXTB groups were intragastrically treated with CXTB at $17.5,35$, and $70 \mathrm{mg} / \mathrm{mL}$, respectively, with $3 \mathrm{~mL} /$ day for 4 weeks (According to Meeh-Rubner formula and direct conversion algorithm, the drug concentration of rats was $35 \mathrm{mg} / \mathrm{mL}$, which was set as mid-dose. The drug concentration of high- and low-dose groups were set as 70 and $17.5 \mathrm{mg} / \mathrm{mL}$ ); meanwhile, the model and control groups were intragastrically treated with $3 \mathrm{~mL}$ normal saline/day for 4 weeks. The dose was calculated by referring to the equivalent human to animal dose ratio according 

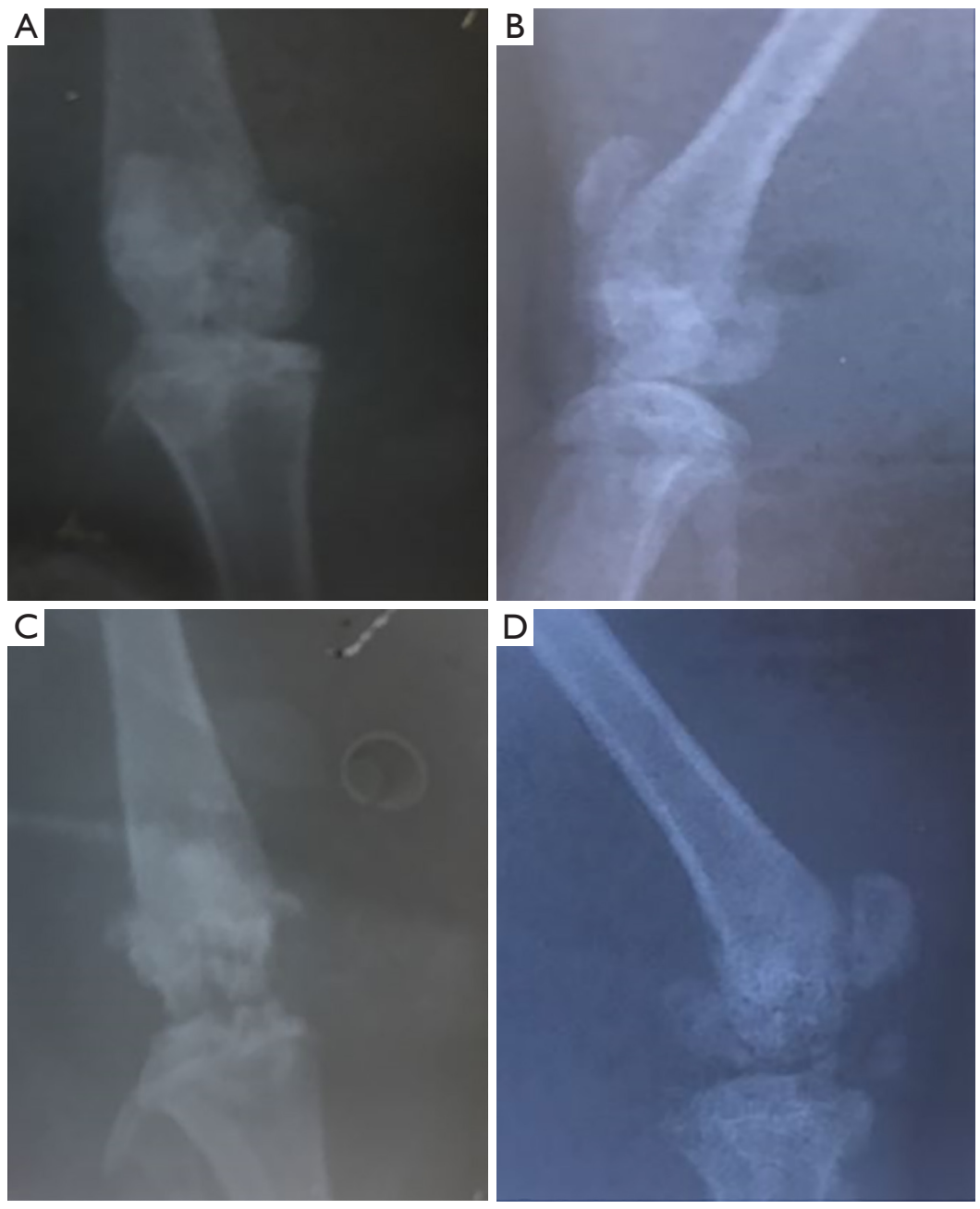

Figure 1 The X-ray film of the knee joint. (A,B) The film of the control group; (C,D) the film after modeling.

to the body surface area. The dose equivalent was $105 \mathrm{mg}$ based on the recommended human dose, which gave a drug concentration of $35 \mathrm{mg} / \mathrm{mL}$ when the daily dose was $3 \mathrm{~mL}$. Then the drug concentration of the high- and low-dose groups was set at 70 and $17.5 \mathrm{mg} / \mathrm{mL}$, respectively. After 4 weeks of administration, all rats were euthanatized with $1 \%$ pentobarbital $(100 \mathrm{mg} / \mathrm{kg})$, the articular cartilages of knee were placed on ice, and the peripheral blood was collected via the abdominal aorta.

\section{Histological observation of cartilage tissue by bematoxylin- eosin staining}

The cartilage tissues were fixed in $4 \%$ paraformaldehyde for $24 \mathrm{~h}$, decalcified in $10 \%$ ethylene diamine tetraacetic acid$2 \mathrm{Na}($ EDTA-2Na), and embedded in paraffin. The tissues were then sectioned $(4 \mu \mathrm{m})$ and stained with hematoxylin and eosin (HE) for histological observation. The cartilage histopathological features were analyzed using the Mankin scoring system. All sections were randomized and evaluated by a trained researcher who was blinded to the five groups.

\section{Western blot}

The tissues were washed with phosphate-buffered saline (PBS), and lysed in lysis buffer on ice for $30 \mathrm{~min}$ to collect protein solution. The concentration of protein was determined with a bicinchoninic acid (BCA) protein assay kit (Beyotime Biotechnology Company, Nanjing, China) according to the manufacturer's protocol. The samples (50 $\mu \mathrm{g}$ protein well) were separated with sodium dodecyl sulfate polyacrylamide gel electrophoresis (SDS-PAGE), and 
transferred to polyvinylidene fluoride (PVDF) membrane after denaturing for $10 \mathrm{~min}$ at $100{ }^{\circ} \mathrm{C}$. The membranes were blocked in 5\% non-fat milk and incubated with the primary antibodies of Rabbit Anti-ADAMTS5 antibody (Abcam Company, Cambridge, UK, \#ab41037) and $\beta$-Actin (Abcam, Cambridge, UK, \#ab8226). Horseradish peroxidaseconjugated secondary antibody (HRP, Abcam, Cambridge, UK, \#ab499900) were added to the membranes for $1 \mathrm{~h}$ at room temperature after washing. The blots were visualized with enhanced chemiluminescence solution (ECL) and a western blot detection system (Image Quant, LAS500, GE Company, Marlborough, MA, USA). Then, the analysis of intensity was measured by Image-Lab software (Bio-Rad Laboratories, Inc., Hercules, CA, USA), and $\beta$-Actin used as an internal control.

\section{RT-PCR}

Total RNA was isolated from cartilage tissues using TRIzol reagent (Invitrogen Corp., Carlsbad, CA, USA) and quantified using a NanoDrop 2000c spectrophotometer (Thermo Fisher Scientific, Waltham, MA, USA). The RNA samples were reverse-transcribed using Roche Reverse Transcriptase Kit (Roche, Basel, Switzerland) in accordance with the manufacturer's instructions. The expression of ADAMTS-5 was determined with $\beta$-actin as control using the $2^{-\Delta \Delta \mathrm{Ct}}$ method. The primers were listed as below: ADAMTS-5: (F: 5'-GGGGTCAGTGTTCTCGCTCTTG-3' and R: 5'-GCCGTTAGGTGGGCAGGGTAT-3'); $\beta$-actin (F: 5'-TGCTATGTTGCCCTAGACTTCG-3' and R: 5'-GCAGTTTTGGGACATTCCTC-3').

\section{ELISA}

The peripheral blood of rats was collected via the abdominal aorta and centrifuged to obtain supernatant serum. The serum was analyzed for inflammatory factors including IL- $1 \beta$ and TNF- $\alpha$ by enzyme-linked immunosorbent assay (ELISA, Beyotime Biotechnology Company, Nanjing, China) according to the manufacturer's instructions.

\section{Statistical analysis}

All data was expressed as mean \pm standard deviation $\left(\bar{x}_{ \pm s}\right)$ and were calculated using one-way analysis of variance (ANOVA) with SPSS version 17.0 software (IBM Corp.,
Armonk, NY, USA). Values were considered significantly different if $\mathrm{P}<0.05$.

\section{Results}

\section{Cartilage tissues were repaired by CXTB in dose-dependent manner}

The histopathologic changes of articular tissues are shown in Figure 2. The HE staining showed that the surface of cartilage in the control group was smooth and clear, the surface cells were small and flat, and the tide line was continuous and complete. In the model group, the cartilage surface was rough, there were cracks and blood vessels passing through cartilage tissue, the 4 layers of structure were confused and illegible, and the tide line lost continuity. In the low-, mid-, and high-dose CXTB groups, the cartilage surface was slightly rough, 4 structural layers were indistinct, the arrangement of chondrocytes was not regular, and degree of cartilage tissue damage was improved compared with the model group.

The Mankin score was used to evaluate the changes of cartilage structure and morphology. The score of the model group was higher than that of other groups $(\mathrm{P}<0.05$, Figure 2), which indicated that the modeling was successful. Conversely, the scores of the low-, mid-, and high-dose CXTB groups decreased sequentially $(\mathrm{P}<0.05$, Figure 2$)$ and were lower than that of the model group $(\mathrm{P}<0.05$, Figure 2$)$. This suggested that CXTB could repair cartilage in a dosedependent manner.

\section{The protein and gene content of ADAMTS-5 was reduced by CXTB in dose-dependent manner}

As shown in Figure 3, the results of western blot showed that the protein content of ADAMTS-5 in the model group was higher than that in other groups $(\mathrm{P}<0.05$, Figure 3). The ADAMTS-5 protein content of the low, mid-, and high-dose CXTB groups decreased in turn $(\mathrm{P}<0.05$, Figure 3). Meanwhile, compared with the model group, ADAMS-5 protein content of the 3 CXTB groups decreased significantly $(\mathrm{P}<0.05$, Figure 3$)$.

The RT-PCR results (Figure 4) showed the gene content of ADAMTS-5. The results and trends were the same as those of western blot.

In general, this suggested that CXTB can reduce protein content and gene expression of ADAMTS-5 in articular cartilage in a dose-dependent manner. 

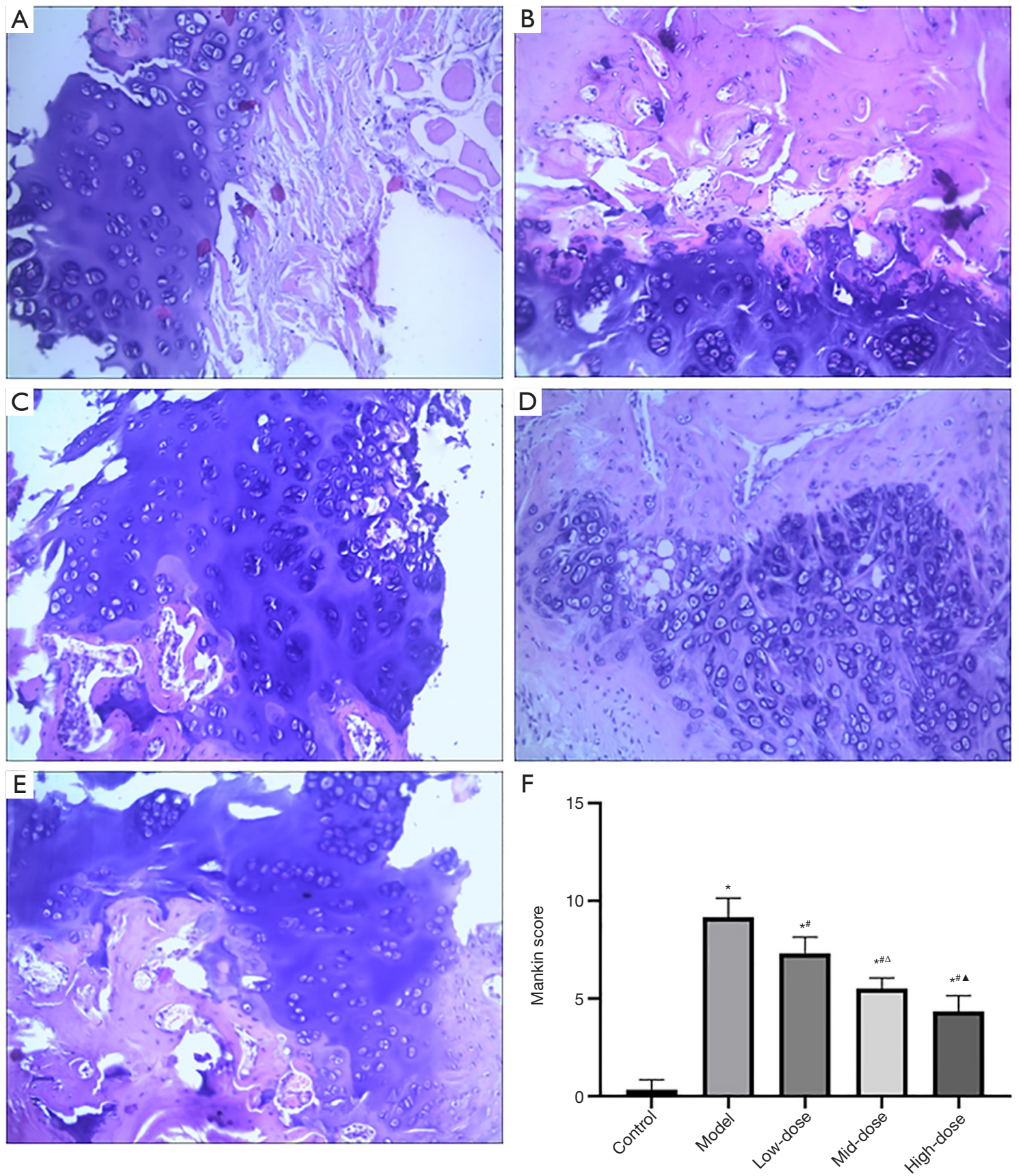

Figure 2 Effects of CXTB on the structure of cartilage tissues. HE staining ( $\times 200)$ and Mankin score of groups. (A) The control group; (B) the model group; (C) the low-dose group; (D) the mid-dose group; (E) the high-dose group; $(\mathrm{F}){ }^{*}, \mathrm{P}<0.05$ vs. the control group; ${ }^{\text {, }} \mathrm{P}<0.05$ vs. the model group; ${ }^{\wedge}, \mathrm{P}<0.05$ vs. the low-dose group; and ${ }^{\wedge}, \mathrm{P}<0.05$ vs. the mid-dose group. CXTB, Cangxitongbi capsule; HE, hematoxylin and eosin. 


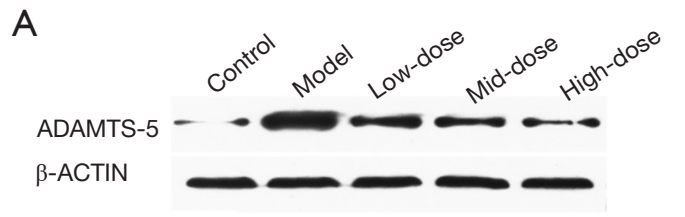

B

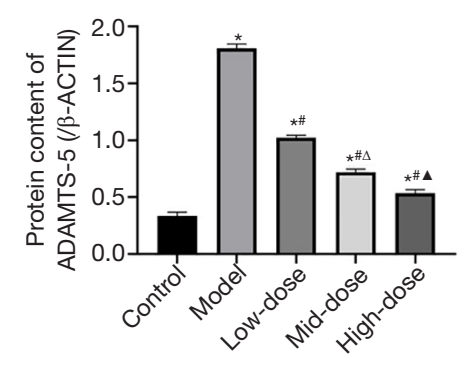

Figure 3 The protein content of ADAMTS-5. (A) Western blot bands of ADAMTS-5. The $\beta$-ACTIN band was selected as control. (B) *, $\mathrm{P}<0.05$ vs. the control group;, $\mathrm{P}<0.05$ vs. the model group; ${ }^{\wedge}, \mathrm{P}<0.05$ vs. the low-dose group; and ${ }^{\wedge}, \mathrm{P}<0.05$ vs. the middose group. ADAMTS-5, a disintegrin and metalloproteinase with thrombospondin motifs- 5 .

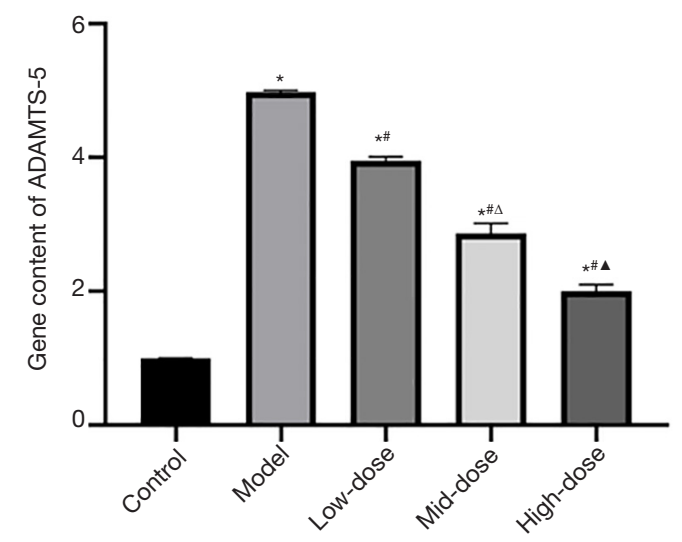

Figure 4 The gene content of ADAMTS-5. * $\mathrm{P}<0.05$ vs. the control group; ${ }^{*}, \mathrm{P}<0.05$ vs. the model group; ${ }^{4}, \mathrm{P}<0.05$ vs. the lowdose group; and $\mathbf{\Delta}, \mathrm{P}<0.05$ vs. the mid-dose group. ADAMTS-5, a disintegrin and metalloproteinase with thrombospondin motifs-5.

\section{CXTB reduced $I L-1 \beta$ and $T N F-\alpha$ in peripheral blood}

The expression levels of inflammatory factors including IL- $1 \beta$ and TNF- $\alpha$ in the serum of rats were measured by ELISA. The results are shown in Figure 5. Compared with the control group, the content of IL- $1 \beta$ and TNF- $\alpha$ in the model group increased significantly $(\mathrm{P}<0.05$, Figure 5). Among the 3 CXTB groups, the contents of IL- $1 \beta$

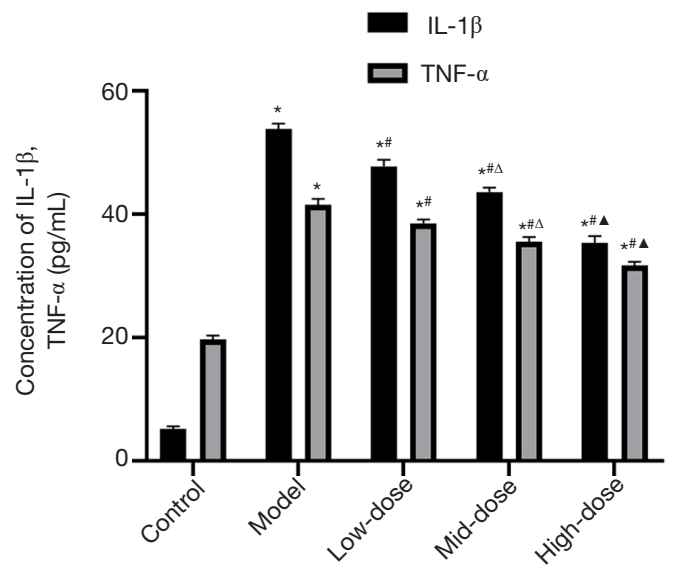

Figure 5 Expression levels of IL-1 $\beta$ and TNF- $\alpha$ in the serum of rats. *, $\mathrm{P}<0.05$ vs. the control group; ${ }^{\#}, \mathrm{P}<0.05$ vs. the model group; ${ }^{\Delta}, \mathrm{P}<0.05$ vs. the low-dose group; and ${ }^{\boldsymbol{}}, \mathrm{P}<0.05$ vs. the mid-dose group. IL-1 $\beta$, interleukin- $1 \beta$; TNF- $\alpha$, tumor necrosis factor- $\alpha$.

and TNF- $\alpha$ were the lowest in the high-dose CXTB group, the second in the mid-dose CXTB group, and the highest in the low-dose CXTB group $(\mathrm{P}<0.05$, Figure 5$)$. The contents of the 3 CXTB groups were lower than those of the model group $(\mathrm{P}<0.05$, Figure 5$)$. This suggested that $\mathrm{CXTB}$ can dose dependently reduce the content of IL- $1 \beta$ and TNF- $\alpha$ in peripheral blood.

\section{Discussion}

The elderly are predominantly affected by the chronic disease that is KOA. It is characterized by the degeneration and loss of knee joint articular cartilage, as well as the new bone formation of the subchondral bone and joint edge. The main symptoms of KOA are joint pain, deformation, and dysfunction. The high incidence has brought a heavy economic and social burden (10-12). At present, there is no effective cure for KOA (13); the main methods include oral nonsteroidal anti-inflammatory drugs and glucosamine sulfate, among others, but these methods have particular disadvantages (14). Increasing attention has been paid to traditional Chinese patent medicines $(15,16)$, which have less side effects. A common traditional Chinese patent medicine for the treatment of KOA, CXTB has predictable clinical effects. However, the mechanism of its treatment of $\mathrm{KOA}$ is not clear. Exploration is required of the detailed mechanism of CXTB to confirm its role in KOA (17). In this study, we established a KOA rat model with the modified Hulth method to explore the effectiveness and 
mechanism of CXTB. The main demonstrations of our study were as follows: (I) damaged cartilage tissue was dose dependently repaired by CXTB; (II) the protein and gene concentration of ADAMTS-5 was reduced in a dosedependent manner by CXTB; (III) the effect of CXTB on ADAMS- 5 reduction may be achieved by reducing IL- $1 \beta$ and TNF- $\alpha$ content.

Proteoglycan is an important component of articular cartilage matrix, which together with collagen network and chondrocytes forms articular cartilage. The progressive loss of proteoglycan is considered to be an important mechanism in the pathogenesis of KOA, with its decline playing a "starter" role. There are 19 kinds of enzymes in the family of ADAMTS, among which ADAMTS-5 is considered to be the most important proteoglycan, which plays an important role in the process of proteolysis $(18,19)$. Previous studies have shown that ADAMTS-5 can hydrolyze the proteoglycan, and cause changes in the microenvironment and mechanical stress distribution of cartilage to stimulate chondrocytes and induce KOA. In our study, CXTB reduced the protein and gene content of ADAMTS-5 in dose-dependent manner, which was consistent with the results of HE staining. It can be inferred that CXTB protects articular cartilage of the knee by down regulating the expression of ADAMTS-5 in a dosedependent manner.

The development of KOA is closely related to inflammation, and IL- $1 \beta$ and TNF- $\alpha$ are the corresponding inflammatory factors $(20,21)$. They are produced by activated synoviocytes, mononuclear cells, or chondrocytes (22), which have been reported to induce apoptosis in chondrocytes and extracellular matrix (ECM) degradation (23). Moreover, previous studies have confirmed that IL- $1 \beta$ and TNF- $\alpha$ increase the content of ADAMTS-5 to induce KOA, thus, they can be seen as the upstream targets of ADAMTS-5 (5-7). In our study, we found that CXTB can down regulate IL- $1 \beta$ and TNF- $\alpha$ in a dose-dependent manner, and the trend is consistent with ADAMTS-5. Based on the above results, we believe that the effect of CXTB on ADAMS-5 reduction may be achieved by reducing the levels of IL- $1 \beta$ and TNF- $\alpha$.

Our study was not without limitations. These experiments were conducted on rats; to achieve a closer indication of possible clinical outcomes for humans, future research using large animals like monkeys is required. Additionally, it is necessary to undertake further study exploring the effective components of CXTB in order to reveal its role of treating KOA.

\section{Conclusions}

In conclusion, CXTB could exert cartilage-protective functions in KOA rat models by down-regulating the production of ADAMTS-5. As upstream targets of ADAMTS-5, IL- $1 \beta$ and TNF- $\alpha$ play roles in regulating ADAMTS-5, and were also down-regulated by CXTB. In all, our study demonstrated that CXTB, as a Chinese patent medicine with minimal side effects, could be a novel therapeutic drug for treating KOA patients.

\section{Acknowledgments}

Funding: This study was supported by the Shandong Province Science and Technology Development Plan of Traditional Chinese Medicine (2017-064) and Jinan City Science and Technology Development Plan (201805044).

\section{Footnote}

Reporting Checklist: The authors have completed the ARRIVE reporting checklist. Available at http://dx.doi. org/10.21037/atm-20-7011

Data Sharing Statement: Available at http://dx.doi. org/10.21037/atm-20-7011

Conflicts of Interest: All authors have completed the ICMJE uniform disclosure form (available at http://dx.doi. org/10.21037/atm-20-7011). The authors have no conflicts of interest to declare.

Ethical Statement: The authors are accountable for all aspects of the work in ensuring that questions related to the accuracy or integrity of any part of the work are appropriately investigated and resolved. The experiments conformed to the guidelines for the care and use of laboratory animals published by the United States National Institutes of Health. All animal experiments were approved by the Animal Ethics Committee of Affiliated Hospital of Shandong University of Traditional Chinese Medicine (AWE-2019-044).

Open Access Statement: This is an Open Access article distributed in accordance with the Creative Commons Attribution-NonCommercial-NoDerivs 4.0 International License (CC BY-NC-ND 4.0), which permits the noncommercial replication and distribution of the article with 
the strict proviso that no changes or edits are made and the original work is properly cited (including links to both the formal publication through the relevant DOI and the license). See: https://creativecommons.org/licenses/by-nc-nd/4.0/.

\section{References}

1. Truong TTT, Lim JM, Cho HR, et al. A DoubleBlind, Randomized Controlled 12-Week Follow-Up Trial to Evaluate the Efficacy and Safety of Polycan in Combination with Glucosamine for the Treatment of Knee Osteoarthritis. Evid Based Complement Alternat Med 2019;2019:9750531.

2. Luo L, Liao M, Peng JX, et al. Comparison of the Efficacy between Conventional Moxibustion and Smoke-Free Moxibustion on Knee Osteoarthritis: A Randomized Controlled Trial. Evid Based Complement Alternat Med 2019;2019:1291947.

3. Wallace IJ, Worthington S, Felson DT, et al. Knee osteoarthritis has doubled in prevalence since the mid20th century. Proc Natl Acad Sci USA 2017;114:9332-6.

4. Murray CJ, Atkinson C, Bhalla K, et al. The state of US health, 1990-2010: burden of diseases, injuries, and risk factors. JAMA 2013;310:591-608.

5. Henrotin Y, Clutterbuck AL, Allaway D, et al. Biological actions of curcumin on articular chondrocytes.

Osteoarthritis Cartilage 2010;18:141-9.

6. Rogerson FM, Chung YM, Deutscher ME, et al. Cytokine-induced increases in ADAMTS-4 messenger RNA expression do not lead to increased aggrecanase activity in ADAMTS-5-deficient mice. Arthritis Rheum 2010;62:3365-73.

7. Gun Bilgic D, Hatipoglu OF, Cigdem S, et al. NF-к $\beta$ upregulates ADAMTS5 expression by direct binding after TNF- $\alpha$ treatment in OUMS-27 chondrosarcoma cell line. Mol Biol Rep 2020;47:4215-23.

8. Wang H, Wang QF, Shi RX, et al. Correlation of genes expression on MyD 88-dependent signaling pathway in progression of knee osteoarthritis. Zhongguo Gu Shang 2018;31:933-6.

9. Wang J, Wang X, Cao Y, et al. Therapeutic potential of hyaluronic acid/chitosan nanoparticles for the delivery of curcuminoid in knee osteoarthritis and an in vitro evaluation in chondrocytes. Int J Mol Med 2018;42:2604-14.

10. Tang X, Wang S, Zhan S, et al. The Prevalence of Symptomatic Knee Osteoarthritis in China: Results From the China Health and Retirement Longitudinal Study. Arthritis Rheumatol 2016;68:648-53.

11. DeRogatis M, Anis HK, Sodhi N, et al. Non-operative treatment options for knee osteoarthritis. Ann Transl Med 2019;7:S245.

12. Plotnikoff R, Karunamuni N, Lytvyak E, et al. Osteoarthritis prevalence and modifiable factors: a population study. BMC Public Health 2015;15:1195.

13. Khan M, Adili A, Winemaker M, et al. Management of osteoarthritis of the knee in younger patients. CMAJ 2018;190:E72-E79.

14. Szerb I, Gal T, Hangody L, et al. Effectiveness evaluation of radiosynovectomy on the radiological progression of osteoarthritis of the knee joint. Eklem Hastalik Cerrahisi 2018;29:147-51.

15. Feng Z, Zheng $W$, Li X, et al. Cryptotanshinone protects against IL-1beta-induced inflammation in human osteoarthritis chondrocytes and ameliorates the progression of osteoarthritis in mice. Int Immunopharmacol 2017;50:161-7.

16. Zheng $\mathrm{W}$, Tao $\mathrm{Z}$, Chen $\mathrm{C}$, et al. Plumbagin prevents IL-1betainduced inflammatory response in human osteoarthritis chondrocytes and prevents the progression of osteoarthritis in mice. Inflammation 2017;40:849-60.

17. Li H, Peng Y, Wang X, et al. Astragaloside inhibits IL-1 $\beta$ induced inflammatory response in human osteoarthritis chondrocytes and ameliorates the progression of osteoarthritis in mice. Immunopharmacol Immunotoxicol 2019;41:497-503.

18. Durigova M, Troeberg L, Nagase H, et al. Involvement of ADAMTS5 and hyaluronidase in aggrecan degradation and release from OSM-stimulated cartilage. Eur Cell Mater 2011,21:31-45.

19. Yang CY, Chanalaris A, Troeberg L. ADAMTS and ADAM metalloproteinases in osteoarthritis - looking beyond the 'usual suspects'. Osteoarthritis Cartilage 2017;25:1000-9.

20. Kapoor M, Martel-Pelletier J, Lajeunesse D, et al. Role of proinflammatory cytokines in the pathophysiology of osteoarthritis. Nat Rev Rheumatol 2011;7:33-42.

21. Santangelo KS, Nuovo GJ, Bertone AL. In vivo reduction or blockade of interleukin-1b in primary osteoarthritis influences expression of mediators implicated in pathogenesis. Osteoarthritis Cartilage 2012;20:1610-8.

22. Xu Y, Dai GJ, Liu Q, et al. Effect of Ermiao Recipe with medicinal guide Angelicae Pubescentis Radix on 
promoting the homing of bone marrow stem cells to treat cartilage damage in osteoarthritis rats. Chin J Integr Med 2014;20:600-9.

23. Sun H, Peng G, Ning X, et al. Emerging roles of long

Cite this article as: Song XY, Xie WP, Zhang P, Zhao M, Bi RX. Cangxitongbi capsule protects articular cartilage of the knee in rats by regulating ADAMTS-5. Ann Transl Med 2020;8(22):1511. doi: 10.21037/atm-20-7011 noncoding RNA in chondrogenesis, osteogenesis, and osteoarthritis. Am J Transl Res 2019;11:16-30.

(English Language Editor: J. Jones) 